\title{
The millimeter-wave spectrum of methyl ketene and the astronomical search for it ${ }^{\star}$
}

\author{
C. Bermúdez ${ }^{1}$, B. Tercero ${ }^{2}$, R. A. Motiyenko ${ }^{1}$, L. Margulès ${ }^{1}$, J. Cernicharo ${ }^{3}$, Y. Ellinger ${ }^{4}$, and J.-C. Guillemin ${ }^{5}$ \\ ${ }^{1}$ Université de Lille, Faculté des Sciences et Technologies, Département Physique, Laboratoire de Physique des Lasers, Atomes et \\ Molécules, UMR CNRS 8523, 59655 Villeneuve d'Ascq Cedex, France \\ e-mail: celina.bermudez@univ-lille1.fr \\ 2 Observatorio Astronómico Nacional (IGN), C/Alfonso XII 3, 28014 Madrid, Spain \\ 3 Instituto de Física Fundamental, Dpt. of Molecular Astrophysics, CSIC, C/ Serrano 121, 28006 Madrid, Spain \\ ${ }^{4}$ Sorbonne Université, CNRS, Laboratoire de Chimie Théorique, LCT, 75005 Paris, France \\ 5 Univ. Rennes, Ecole Nationale Supérieure de Chimie de Rennes, CNRS, ISCR - UMR6226, 35000 Rennes, France
}

Received 19 April 2018 / Accepted 11 July 2018

\begin{abstract}
Context. The analysis of isomeric species of a compound observed in the interstellar medium (ISM) is a useful tool to understand the chemistry of complex organic molecules. It could, likewise, assist in the detection of new species.

Aims. Our goal consists in analyzing one of the two most stable species of the $\mathrm{C}_{3} \mathrm{H}_{4} \mathrm{O}$ family, methyl ketene, whose actual rotational parameters are not precise enough to allow its detection in the ISM. The obtained parameters will be used to search for it in the highmass star-forming regions Orion KL and Sagittarius B2, as well as in the cold dark clouds TMC-1 in the Taurus Molecular Cloud and Barnard 1 (B1-b).

Methods. A millimeter-wave room-temperature rotational spectrum of methyl ketene was recorded from 50 to $330 \mathrm{GHz}$. The internal rotation analysis of its ground state and first torsional excited state was performed with the rho-axis method employing the RAM36 program.

Results. More than 3000 transitions of the rotational spectrum of the ground state $\left(K_{\mathrm{a}} \mathrm{max}=18\right)$ and first torsional excited state $\left(K_{\mathrm{a}} \max =13\right)$ of methyl ketene were fitted using a Hamiltonian that contains 41 parameters with a root mean square of $44 \mathrm{kHz}$. Column density limits were calculated but no lines were detected in the ISM belonging to methyl ketene.
\end{abstract}

Key words. ISM: molecules - methods: laboratory: molecular - submillimeter: ISM - molecular data - line: identification

\section{Introduction}

A fundamental question in astrophysics and astrochemistry yet to be answered is to understand molecular complexity in space. As a result of vast scientific efforts, 200 different chemical species have been detected in the interstellar medium (ISM). Among them, one can find several complex organic molecules (COMs) under diverse environments, meaning by COMs carbon-based molecular species with more than six atoms in their structure (Herbst \& van Dishoeck 2009). COMs up to 13 atoms have been detected in the ISM containing a large variety of functional groups, including alcohols, ketones, or aldehydes (Endres et al. 2016). The most abundant sources of these COMs are the giant molecular clouds of the Galactic Center (Requena-Torres et al. 2006; Hollis et al. 2006b) as well as high-mass hot cores (Ikeda et al. 2001; Remijan et al. 2004; Belloche et al. 2013; Tercero et al. 2013, 2015) and lowmass hot corinos (Bottinelli et al. 2007; Jørgensen et al. 2016) of star-forming regions. However, the detection of COMs has been recently extended to dark cloud cores and pre-stellar cores (see, e.g., Cernicharo et al. 2012; Jiménez-Serra et al. 2016; McGuire et al. 2018).

\footnotetext{
* Full Tables 2 and 3 are only available at the CDS via anonymous ftp to cdsarc.u-strasbg. fr (130.79.128.5) or via http://cdsarc. u-strasbg.fr/viz-bin/qcat?J/A+A/619/A92
}

Notwithstanding the advances in this field, the synthetic routes of COMs are still far from being fully understood; the creation of reliable models is particularly challenging in sources like Sgr B2(N) (Quan \& Herbst 2007), which, is one of the major sources of COMs. Hollis et al. (2006a) pointed out that the observation of molecular isomers provides an excellent tool to evaluate the hypothesis of synthetic pathways. More recently, after evaluating several groups of molecular isomers, Lattelais et al. $(2009,2010,2011)$ proposed the "minimum energy principle" (MEP). They stated that the thermodynamically most stable isomer of a molecular formula is practically the most abundant in the ISM. If so, this principle would constitute a pragmatic tool to propose new candidates for the ISM.

Continuing the isomeric analysis, in the present paper we will focus on the $\mathrm{C}_{3} \mathrm{H}_{4} \mathrm{O}$ family. The most stable isomers $\mathrm{C}_{3} \mathrm{H}_{4} \mathrm{O}$ are propenal (also known as acrolein), methyl ketene (1-propen1-one), and cyclopropanone (Karton \& Talbi 2014). So far, only a tentative detection of propenal has been reported towards Sgr B2(N) employing the Green Bank telescope (Hollis et al. 2004), where they observed the two transitions that were in the observational frequency range but the detection of propenal has never been confirmed by independent observations. A more recent work in the millimeter frequency range (Daly et al. 2015) provides a comprehensive spectroscopic analysis that could allow us to detect further transitions. Nevertheless, the main issue of the analysis of this group of isomers is the lack of precise 


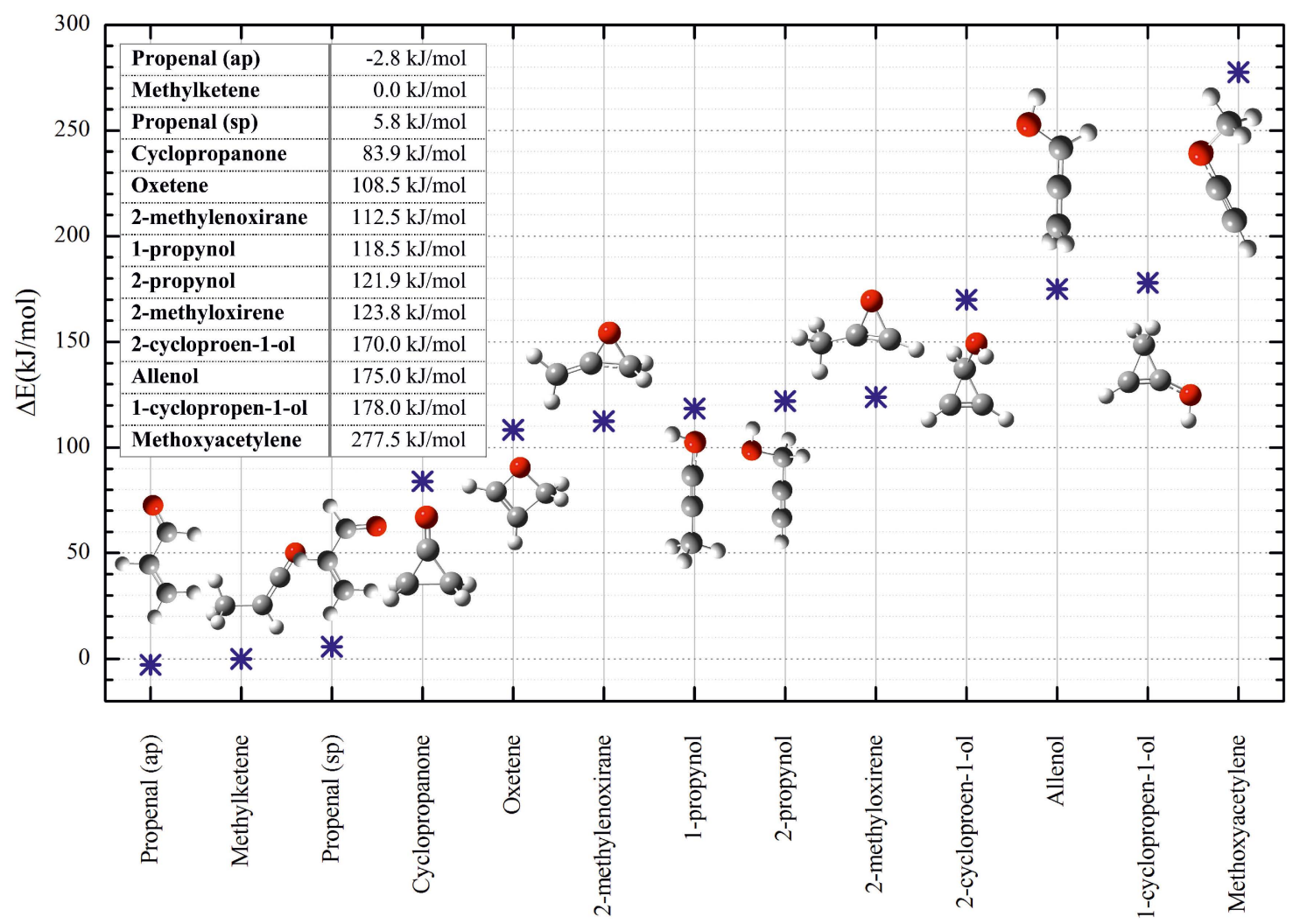

Fig. 1. Relative energy of $\mathrm{C}_{3} \mathrm{H}_{4} \mathrm{O}$ isomers calculated at $\mathrm{CCSD}(\mathrm{T}) /$ aug-cc-pVTZ level of theory.

information about the spectroscopy of methyl ketene, which prevents not only its detection in the ISM but also the development of models that explain the behavior of the isomeric $\mathrm{C}_{3} \mathrm{H}_{4} \mathrm{O}$ family in the ISM. The latest rotational parameters for methyl ketene were published by Bak et al. $(1962,1966)$ in the 1960 s where they reported the analysis up to $J=3$ and $K_{\mathrm{a}}=2$ in the $16-27 \mathrm{GHz}$ frequency range. These values do not allow a precise extrapolation to the ISM observational frequency range. Since its homologue, ketene $\left(\mathrm{H}_{2} \mathrm{CCO}\right)$, was detected a long time ago (Turner 1977) and provided that its isomer acrolein is also likely to be present in the ISM, we investigate the rotational spectrum of methyl ketene in order to facilitate its detection in the ISM.

\section{Computational calculations}

Our own high level ab initio calculations were performed with the aim of comparing the energetic ratios between the lower energy $\mathrm{C}_{3} \mathrm{H}_{4} \mathrm{O}$ isomers: propenal (antiperiplanar rotamer, ap), methyl ketene, propenal (synperiplanar rotamer, sp), cyclopropanone, oxetene, 2-methyleneoxirane, 1-propynol, 2-propynol, 2-methyloxirene, 2-cyclopropen-1-ol, 1-cyclopropen-1-ol, allenol, and methoxyacetylene. The coupled cluster method CCSD(T) was employed together with Dunning basis sets aug-cc-pVTZ as implemented in MOLPRO software (Werner et al. 2015). A summary of the results is presented in Fig. 1. Three species, methyl ketene and both rotamers of propenal, were found to be the most stable, around $80 \mathrm{~kJ} \mathrm{~mol}^{-1}$ below cyclopropanone, the following isomer on the energy scale.

Within the three most stable species, methyl ketene is the only one whose spectroscopic parameters that could allow its detection in the ISM remain unknown. In order to assist the spectroscopic analysis of methyl ketene, anharmonic force field calculations were carried out (Frisch et al. 2016) at the
MP2/aug-cc-pVTZ level of theory. The complete set of rotational constants, distortional constants, structure, and internal rotation parameters, including the barrier height, can be found in Table A.1. The $\mathrm{CH}_{3}$ internal rotation barrier height is estimated at $424 \mathrm{~cm}^{-1}$, which is in a good agreement with the value of $411 \pm 7 \mathrm{~cm}^{-1}$ obtained by Bak et al. (1966).

At the MP2/aug-cc-pVTZ level of theory, the calculations of vibrational excited states' energies establish that methyl ketene has two low-frequency vibrational modes: the methyl group torsion $\left(v_{18}=1, A^{\prime \prime}\right)$ that has a fundamental frequency of $133.4 \mathrm{~cm}^{-1}$, and skeletal in-plane bending in $\left(v_{12}=1, A^{\prime}\right)$ at $202 \mathrm{~cm}^{-1}$. The value obtained for $v_{18}=1$ is consistent with the corresponding value estimated by Winther et al. (2002) in the infrared (IR) experiments $\left(135.6 \pm 1 \mathrm{~cm}^{-1}\right)$.

\section{Experiments}

The methyl ketene sample was obtained by pyrolysis of propionic anhydride, which was purchased from Aldrich and used without further purification. Flash vacuum pyrolysis at $600^{\circ} \mathrm{C}$ produces methyl ketene together with propanoic acid. The latter was easily removed by selective condensation in a trap immersed in a $-70^{\circ} \mathrm{C}$ cold bath. A gaseous flow of pure methyl ketene was thus obtained and analyzed by means of millimeter-wave spectroscopy.

The millimeter-wave spectrum of methyl ketene has been recorded between $50-330 \mathrm{GHz}$ at room temperature and at a pressure of $0.028 \mathrm{mbar}$ using the Lille spectrometer described by Zakharenko et al. (2015), which was recently equipped with a fast-scan mode. This new fast-scan design is able to provide a rapid frequency scan with up to $50 \mu \mathrm{s} /$ point-of-frequency switching rate. It employs an AD9915 direct digital synthesizer (DDS) up-converted into $\mathrm{Ku}$ band. The up-conversion is achieved by 


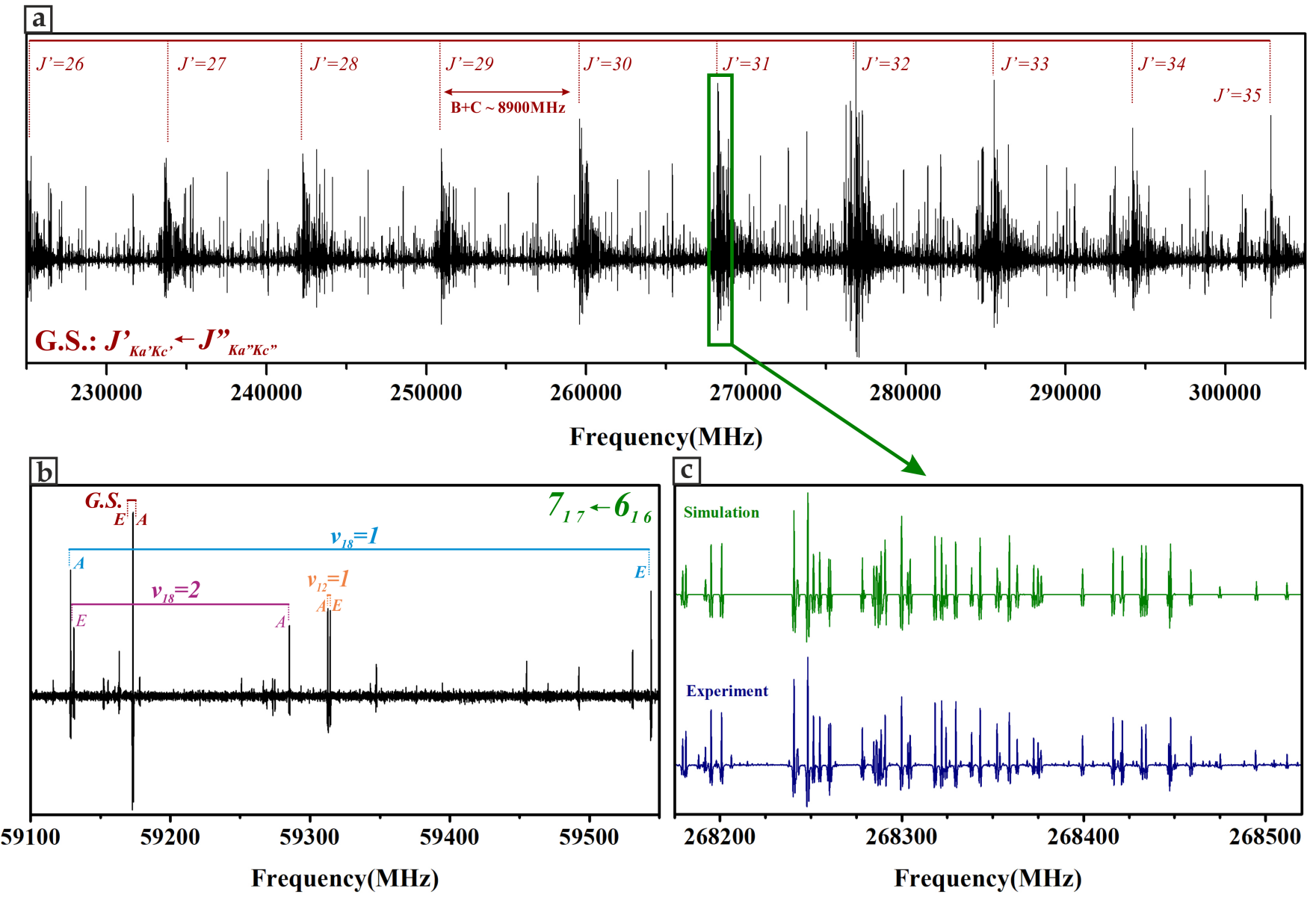

Fig. 2. Sections of the methyl ketene rotational spectrum.

mixing the DDS output signal in the frequency range 320$420 \mathrm{MHz}$, and the signal from an Agilent E8257 synthesizer (up to $20 \mathrm{GHz}$ ) with subsequent filtering of the upper sideband in a YIG bandpass filter. Thus, the fast frequency scan is provided by sweeping the DDS within the filter bandwidth of $25-40 \mathrm{MHz}$. The up-converted signal is then multiplied by factor of six into $W$-band $(75-110 \mathrm{GHz})$ using an active multiplier AMC-10 from VDI (Virginia Diodes, Inc). The output power of AMC-10 is enough to drive passive Schottky multipliers from VDI on the next stage of frequency multiplication: a doubler $(150-220 \mathrm{GHz})$ and a tripler $(225-330 \mathrm{GHz})$. In addition, AMC-10 may be used as a radiation source in $W$-band. The detection was carried out employing a series of Schottky diode zero biased detectors from VDI: WR10ZBD (75-110 GHz), WR5.1ZBD (150-220 GHz), and WR3.4ZBD $(225-330 \mathrm{GHz})$. The $50-75 \mathrm{GHz}$ range was covered employing a Millitech active multiplier by factor four and the corresponding solid state detector WR15ZBD.

\section{Analysis of the spectra}

Methyl ketene is a near prolate molecule that has a planar molecular skeleton with only two hydrogen atoms of the methyl group out of the plane, thus it has $C_{\mathrm{s}}$ species at equilibrium. Due to the coupling of internal rotation of the methyl group $\left(\mathrm{CH}_{3}\right)$ and the overall rotation of the molecule, the rotational levels are split into $\mathcal{A} / \mathcal{E}$. One should note that methyl ketene is characterized by a relatively high value of the $\rho$ parameter $(\rho=0.196)$ that describes this coupling. The $\rho$ parameter corresponds to the norm of the so-called $\rho$ vector, whose coordinates are calculated using the expression

$\rho_{g}=\frac{\lambda_{g} I_{\alpha}}{I_{g}},(g=x, y, z)$,

where $\lambda_{g}$ are the direction cosines of the internal rotation axis of the top in the principal axis system, $I_{g}$ are the principal moments of inertia, and $I_{\alpha}$ is the moment of inertia of the methyl top.

In terms of the $\rho$ value, methyl ketene may be considered as an intermediate case between acetaldehyde $\mathrm{CH}_{3} \mathrm{CHO} \rho=0.328$ (Smirnov et al. 2014) and acetic acid $\mathrm{CH}_{3} \mathrm{COOH} \rho=0.072$ (Ilyushin et al. 2013) or methyl formate $\mathrm{CH}_{3} \mathrm{OCHO}, \rho=0.084$ (Ilyushin et al. 2009). For all these molecules, accurate analysis of the large-amplitude torsional motion was performed using the rho-axis method (RAM). Therefore, RAM was applied in the present study of methyl ketene. The method uses the axis system obtained by rotation of the principal axis system to align with the new $z$ axis parallel to the $\rho$ vector. For prolate rotors with a plane of symmetry, the new axis system is obtained via rotation of the principal axes in the $(a b)$ plane by angle $\theta_{\mathrm{RAM}}$ :

$\theta_{\mathrm{RAM}}=\arctan \left(\frac{\rho_{\mathrm{b}}}{\rho_{\mathrm{a}}}\right)$

The RAM Hamiltonian may be written as (Kleiner 2010)

$H_{\mathrm{RAM}}=H_{\mathrm{T}}+H_{\mathrm{R}}+H_{\mathrm{cd}}+H_{\text {int }}$, 
where $H_{\mathrm{T}}$ represents the torsional Hamiltonian defined as

$H_{\mathrm{T}}=F\left(p_{\alpha}-\rho J_{\mathrm{z}}\right)^{2}+V(\alpha)$,

where $F$ is the internal rotation constant, $p_{\alpha}$ is the internal rotation angular momentum conjugate to the torsion angle $\alpha$, and $V(\alpha)$ is the internal rotation potential function expanded into

$$
V(\alpha)=\frac{1}{2} V_{3}(1-\cos 3 \alpha)+\frac{1}{2} V_{6}(1-\cos 6 \alpha)+\ldots
$$

The $H_{\mathrm{R}}$ represents rigid rotor rotational Hamiltonian in the rho-axis system. In addition to the usual $A, B$, and $C$ terms, for the molecules with a plane of symmetry $(\chi z)$ (as is appropriate for methyl ketene) it contains a non-diagonal term $D_{\mathrm{xz}}$. In the $I^{\mathrm{r}}$ coordinate representation, $H_{\mathrm{R}}$ has the form

$H_{\mathrm{R}}=A_{\mathrm{RAM}} J_{z}^{2}+B_{\mathrm{RAM}} J_{x}^{2}+C_{\mathrm{RAM}} J_{y}^{2}+D_{x z}\left(J_{x} J_{z}+J_{z} J_{x}\right)$.

The last two terms in the Eq. (1), $H_{\text {cd }}$ and $H_{\text {int }}$, are the usual centrifugal distortion and higher-order torsional-rotational interaction Hamiltonians, respectively.

For fitting and predicting the rotational spectra, we used the RAM36 (rho-axis method for three- and six-fold barriers) program that allows us to include in the model almost any symmetry-allowed torsion-rotation Hamiltonian term up to the twelfth order (Ilyushin et al. 2010, 2013). The RAM Hamiltonian in the Eq. (1) may be expressed in the following form used in the RAM36 program:

$$
\begin{aligned}
H=\frac{1}{2} & \sum_{k n p q r s} B_{\text {knpqrs } 0}\left[J^{2 k} J_{z}^{n} J_{x}^{p} J_{y}^{q} p_{\alpha}^{r} \cos (3 s \alpha)\right. \\
& \left.+\cos (3 s \alpha) p_{\alpha}^{r} J_{y}^{q} J_{x}^{p} J_{z}^{n} J^{2 k}\right] \\
& +\frac{1}{2} \sum_{k n p q r t} B_{k n p q r 0 t}\left[J^{2 k} J_{z}^{n} J_{x}^{p} J_{y}^{q} p_{\alpha}^{r} \sin (3 t \alpha)\right. \\
& \left.+\sin (3 t \alpha) p_{\alpha}^{r} J_{y}^{q} J_{x}^{p} J_{z}^{n} J^{2 k}\right]
\end{aligned}
$$

where the $B_{\text {knpqrst }}$ are fitting parameters. Methyl ketene is characterized as a molecule having a $C_{\mathrm{s}}$ frame and a $C_{3 \mathrm{v}}$ top. Therefore the allowed terms in the torsion-rotation Hamiltonian must be totally symmetric in the molecular symmetry group $G_{6}$ (and also must be Hermitian and invariant to the time reversal operation). Since all individual operators $p_{\alpha}, J_{x}, J_{y}, J_{z}, J^{2}, \cos (3 s \alpha)$, and $\sin (3 t \alpha)$ used in Eq. (5) are Hermitian, all possible terms provided by Eq. (5) will automatically be Hermitian. The particular term to be fit is represented in the input file with a set of $k, n, p, q, r, s, t$ integer indices that are checked by the program for conformity with time reversal and symmetry requirements to prevent accidental introduction of symmetry-forbidden terms into the Hamiltonian. For example, $B_{0200000}$ corresponds to $A_{\mathrm{RAM}}$ in Eq. (4), $B_{0000200}$ to $F$ in Eq. (2), $B_{0110000}$ to $2 D_{\text {ab }}$ in Eq. (4), and so on. In Table 1 , which presents the final set of molecular parameters, instead of $B_{\text {knpqrst }}$, we give more conventional names for the parameters whose nomenclature is based on the subscript procedures of Xu et al. (2008). Further details on the Hamiltonian employed can be seen from the operators presented in Table 1.

We started our analysis of methyl ketene by refitting 16 transitions involving low $J$ and $K_{\text {a }}$ quantum numbers reported by Bak et al. $(1962,1966)$ using the RAM36 code. The RAM model is highly non-linear and strongly depends on the dataset of assigned transitions. In the initial fit, owing to the limited number of transitions from previous studies, the $F$ and $\rho$ parameters

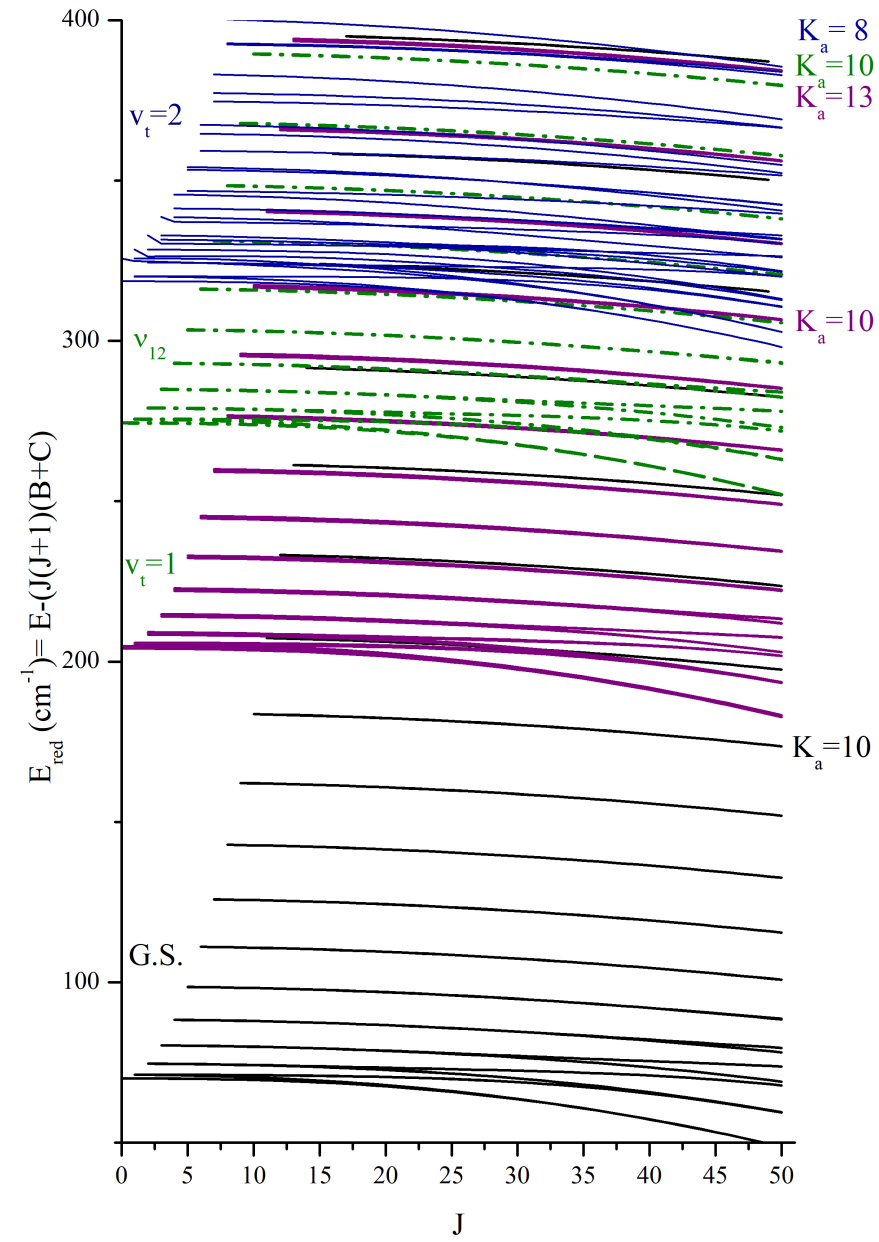

Fig. 3. Reduced energy diagram where the reduced energy of each rotational state of the different vibrational states is represented in function of the quantum number $J$. The reduced energies $E_{\text {red }}$ are calculated as $E_{\text {red }}\left(\mathrm{cm}^{-1}\right)=E-(J(J+1)(B+C))$ employing the energies obtained from the fit for the torsional states and the corrected ab initio values for $v_{12}$.

from the torsional Hamiltonian (Eq. (2)) were fixed to the values determined from the $r_{\mathrm{s}}$ structure of methyl ketene (Bak et al. 1966). Following the fit of previously reported transitions, initial frequency predictions were obtained. Not surprisingly, the predictions could only be reliable for the lower frequency range and low values of $J$ and $K_{\mathrm{a}}$. For example, the analysis of the data of Bak et al. $(1962,1966)$ predicts $\mathcal{A}$ symmetry transition $25_{025} \leftarrow 24_{024}$ at $210496.3 \mathrm{MHz}$, whereas the experimental value is $210746.8 \mathrm{MHz}$, which differs by about $250 \mathrm{MHz}$. Nevertheless, the initial predictions were useful in the assignment of millimeter-wave spectra.

Figure $2 \mathrm{a}$, represents the experimental spectrum of methyl ketene in the frequency range $225-305 \mathrm{GHz}$ measured in this study. At first glance, groups of ${ }^{a} R_{0,1}$ transitions separated by $\sim 8900 \mathrm{MHz}$ may be observed. The separation between groups corresponds approximately to the value of $B+C$ for an $a$-type spectrum of a quasi prolate molecule like methyl ketene, and it provides the assignment of the $J$ quantum number within each group. In this way, using the results of our theoretical calculations, predictions obtained on the basis of the refit results of previous studies, and broadband spectra suitable for building Loomis-Wood diagrams, we iteratively assigned and analyzed the rotational spectrum of methyl ketene. 
Table 1. Methyl ketene molecular parameters of ground state and torsional mode $v_{18}$ obtained from the fit using RAM36 program $(A-$ reduction, $\mathcal{I}^{r}$-representation).

\begin{tabular}{|c|c|c|c|}
\hline Parameter $^{a}$ & Operator $^{b}$ & $n_{\mathrm{op}} t_{\mathrm{op}} r_{\mathrm{op}}{ }^{c}$ & Values $(\mathrm{MHz})^{d}$ \\
\hline$A_{\mathrm{RAM}}$ & $J_{\mathrm{a}}^{2}$ & $2_{0,2}$ & $38587.00(11)$ \\
\hline$B_{\mathrm{RAM}}$ & $J_{\mathrm{b}}^{2}$ & 20,2 & $4773.813(29)$ \\
\hline$C_{\mathrm{RAM}}$ & $J_{\mathrm{c}}^{0}$ & 20,2 & $4139.199(46)$ \\
\hline$D_{\mathrm{ab}}$ & $\left\{J_{\mathrm{a}}, J_{\mathrm{b}}\right\}$ & 20,2 & $-3015.26(39)$ \\
\hline$\rho^{e}$ & $p_{\alpha} J_{\mathrm{a}}$ & $22_{1,1}$ & $0.194354(13)^{e}$ \\
\hline$F^{f}$ & $p_{\alpha}^{2}$ & 22,0 & $6.4215(13)^{\mathrm{f}}$ \\
\hline$V_{3}^{f}$ & $1 / 2(1-\cos 3 \alpha)$ & 2,0 & $429.38(11)^{\mathrm{f}}$ \\
\hline$\Delta_{\mathrm{J}}$ & $-J^{4}$ & $4_{0,4}$ & $0.46682(81) 10^{-2}$ \\
\hline$\Delta_{\mathrm{JK}}$ & $-J^{2} J_{\mathrm{a}}^{2}$ & $4_{0,4}$ & $-0.17168(62) 10^{0}$ \\
\hline$\Delta_{\mathrm{K}}$ & $-J_{\mathrm{a}}^{4}$ & $4_{0,4}$ & $0.47291(60) 10^{1}$ \\
\hline$\delta_{\mathrm{J}}$ & $-2 J^{2}\left(J_{\mathrm{b}}^{2}-J_{\mathrm{c}}^{2}\right)$ & $4_{0,4}$ & $0.15904(40) 10^{-2}$ \\
\hline$\delta_{\mathrm{K}}$ & $-2\left\{J_{\mathrm{a}}^{2},\left(J_{\mathrm{b}}^{2}-J_{\mathrm{c}}^{2}\right)\right\}$ & $4_{0,4}$ & $0.2120(19) 10^{-1}$ \\
\hline$D_{\text {abJ }}$ & $\left\{J_{\mathrm{a}}, J_{\mathrm{b}}\right\} J^{2}$ & $4_{0,4}$ & $0.3983(19) 10^{-1}$ \\
\hline$D_{\mathrm{abK}}$ & $\left\{J_{\mathrm{a}}, J_{\mathrm{b}}\right\} J_{\mathrm{a}}^{2}$ & $4_{0,4}$ & $0.5329(38) 10^{0}$ \\
\hline$\rho_{\mathrm{J}}$ & $p_{\alpha} J_{\mathrm{a}} J^{2}$ & $4_{1,3}$ & $-0.4253(14) 10^{0}$ \\
\hline$\rho_{\mathrm{K}}$ & $p_{\alpha} J_{\mathrm{a}}^{3}$ & $4_{1,3}$ & $0.21648(59) 10^{2}$ \\
\hline$F_{\mathrm{J}}$ & $p_{\alpha}^{2} J^{2}$ & $4_{2,2}$ & $0.15159(31) 10^{0}$ \\
\hline$F_{\mathrm{K}}$ & $p_{\alpha}^{2} J_{\mathrm{a}}^{2}$ & $4_{2,2}$ & $-0.4535(31) 10^{2}$ \\
\hline$F_{\mathrm{ab}}$ & $1 / 2\left\{J_{\mathrm{a}}, J_{\mathrm{b}}\right\} p_{\alpha}^{2}$ & $4_{2,2}^{2,2}$ & $0.1738(68) 10^{1}$ \\
\hline$V_{3 \mathrm{~J}}$ & $J^{2}(1-\cos 3 \alpha)$ & $4_{2,2}$ & $-0.13759(47) 10^{2}$ \\
\hline$V_{3 \mathrm{~K}}$ & $J_{\mathrm{a}}^{2}(1-\cos 3 \alpha)$ & $4_{2,2}$ & $-0.1229(28) 10^{3}$ \\
\hline$V_{3 \mathrm{ab}}$ & $1 / 2\left(\left\{J_{\mathrm{a}}, J_{\mathrm{b}}\right\}\right)(1-\cos 3 \alpha)$ & $4_{2,2}$ & $-0.1318(30) 10^{3}$ \\
\hline$V_{3 \mathrm{bc}}$ & $\left(J_{\mathrm{b}}^{2}-J_{\mathrm{c}}^{2}\right)(1-\cos 3 \alpha)$ & $4_{2,2}$ & $0.2134(47) 10^{1}$ \\
\hline$D_{3 \mathrm{ac}}$ & ${ }^{1} / 2\left\{J_{\mathrm{a}}, J_{\mathrm{c}}\right\} \sin 3 \alpha$ & $4_{2,2}$ & $0.840(10) 10^{3}$ \\
\hline$\rho_{\mathrm{m}}$ & $p_{\alpha}^{3} J_{\mathrm{a}}$ & $4_{1,3}$ & $0.514(11) 10^{2}$ \\
\hline$F_{\mathrm{m}}$ & $p_{\alpha}^{4}$ & $4_{4,0}$ & $-0.443(15) 10^{2}$ \\
\hline$V_{6}$ & $1 / 2(1-\cos 6 \alpha)$ & $4_{4,0}$ & $-0.3088(44) 10^{6}$ \\
\hline$\Phi_{\mathrm{J}}$ & $J^{6}$ & $6_{0,6}$ & $0.21428(94) 10^{-7}$ \\
\hline$\Phi_{\mathrm{JKK}}$ & $J^{2} J_{\mathrm{a}}^{4}$ & $6_{0,6}$ & $-0.7678(62) 10^{-4}$ \\
\hline$\Phi_{\mathrm{K}}$ & $J_{\mathrm{a}}^{6}$ & $6_{0,6}$ & $0.8161(75) 10^{-3}$ \\
\hline$\phi_{\mathrm{J}}$ & $2 J^{4}\left(J_{\mathrm{b}}^{2}-J_{\mathrm{c}}^{2}\right)$ & $6_{0,6}$ & $0.9235(85) 10^{-8}$ \\
\hline$\rho_{\mathrm{JK}}$ & $p_{\alpha} J_{\mathrm{a}}^{3} J^{2}$ & $6_{1,5}$ & $0.5936(53) 10^{-3}$ \\
\hline$\rho_{\mathrm{KK}}$ & $p_{\alpha} J_{\mathrm{a}}^{5}$ & $6_{1,5}$ & $-0.6917(54) 10^{-2}$ \\
\hline$F_{\mathrm{JK}}$ & $p_{\alpha}^{2} J^{2} J_{\mathrm{a}}^{2}$ & 62,4 & $-0.1522(14) 10^{-2}$ \\
\hline$F_{\mathrm{KK}}$ & $p_{\alpha}^{2} J_{\mathrm{a}}^{4}$ & $6_{2,4}$ & $0.1585(15) 10^{-1}$ \\
\hline$V_{3 \mathrm{JK}}$ & $J^{2} J_{\mathrm{a}}^{2}(1-\cos 3 \alpha)$ & 62,4 & $0.1922(51) 10^{-1}$ \\
\hline$\rho_{\mathrm{mbc}}$ & $p_{\alpha}^{3} J_{\mathrm{a}}\left(J_{\mathrm{b}}^{2}-J_{\mathrm{c}}^{2}\right)$ & 6,3 & $0.677(15) 10^{-3}$ \\
\hline$V_{6 \mathrm{ab}}$ & $\left\{J_{\mathrm{a}}, J_{\mathrm{b}}\right\}(1-\cos 6 \alpha)$ & $6_{4,2}$ & $0.425(10) 10^{2}$ \\
\hline$\sigma_{\text {rms/wrms }}{ }^{g}$ & & & $4.4210^{-2} / 0.74$ \\
\hline$J_{\max } / K_{\mathrm{a}, \max }{ }^{h}$ & & & $38 / 18$ \\
\hline$N_{\text {transit }} / N_{\text {blenc }}$ & & & $3055 / 474$ \\
\hline
\end{tabular}

Notes. ${ }^{(a)}$ Parameter nomenclature. ${ }^{(b)}$ Torsional-rotation operators employed in the model in the $I^{r}$ representation. ${ }^{(c)} n_{\mathrm{op}}$ is the total order operator which is $n_{\mathrm{op}}=t_{\mathrm{op}}+r_{\mathrm{op}}$, being $t_{\mathrm{op}}$ the order of the torsional part, and $r_{\mathrm{op}}$ that of the rotational part. ${ }^{(d)}$ Unless it is indicated, all constants are expressed in MHz. ${ }^{(e)} \rho$ value is unitless. ${ }^{(f)} F$ and $V_{3}$ values are expressed in $\mathrm{cm}^{-1}$. ${ }^{(g)}$ Fit root mean squared error in $\mathrm{MHz} /$ weighted root mean squared error. ${ }^{\left({ }^{h}\right)}$ Maximum value of quantum number $J$ and $K_{\mathrm{a}}$ included in the fit. ${ }^{(i)}$ Number of transitions included in the fit/Number of blended transitions.

The advantage of the RAM is its capacity for a global treatment of all excited vibrational states associated with the methyl torsion. Using the results of the fit of the ground-state transitions, we were able to predict the transitions of the $v_{18}=1$ state within a few $\mathrm{MHz}$. In the same manner, we were able to obtain rather accurate predictions for the $v_{18}=2$ state on the basis of the results of the global fit of the $v=0$ and $v_{18}=1$ states. Looking closely at the spectra (see Fig. 2b), we observed several satellites around the ground state, which belong to vibrational excited states. After scaling the rotational constants of the vibrational excited states by a correction factor obtained from the experimental and calculated ground-state values, we were able to assign several series of rotational transitions of the $v_{12}=1$ state.

The global fit of the $v_{18}=0,1$, and 2 states was a very lengthy task as it required a much higher number of parameters that led to strong correlations, and unrealistic values of the main torsional parameters such as $F, V_{3}$, and $\rho$. To address this issue, we built a reduced energy diagram presented in Fig. 3 . The diagram represents the reduced energy $E_{\text {red }}$ of each rotational state as a function of $J$ quantum number. It is calculated according to the equation $E_{\mathrm{red}}\left(\mathrm{cm}^{-1}\right)=E-J(J+1)(B+C)$, where $E$ corresponds to the energy of rotational level predicted either on the basis of global fit or quantum chemical calculations. Each line on the diagram represents a stack of levels with the same $K_{\mathrm{a}}$ quantum number. The analysis of the diagram shows that starting from the energy of $300 \mathrm{~cm}^{-1}$ the density of levels is significantly increased. For example, around $400 \mathrm{~cm}^{-1}$ the rotational levels of different vibrational states with close $K_{\mathrm{a}}$ values are embedded into a narrow energy zone. Thus, taking into account the proximity in energy of $v_{18}=2$ and $v_{12}=1$ and their symmetry (both $A^{\prime}$ ), $c$-type Coriolis and Fermi interactions are expected to occur, even at low $K_{\mathrm{a}}$ transitions, which cannot be taken into consideration employing the actual RAM Hamiltonian. Consequently we prefer to exclude the $v_{18}=2$ state from consideration and to provide the analysis of the ground and $v_{18}=1$ states.

The complete dataset treated at the final stage of the current study contains $3055 a$ - and $b$-type transitions (where 474 are blended), including 1844 transitions of the ground state and 1211 transitions of the $v_{18}=1$ state. The maximum values of $K_{\mathrm{a}}$ for the ground and $v_{18}=1$ states are 18 and 12 , respectively. All these rotational levels have a reduced energy lower than $400 \mathrm{~cm}^{-1}$, thus right below the energy of the $V_{3}$ barrier. The final fit uses a model consisting of 38 parameters that reproduced the experimental data with a weighted root-mean-square deviation of 0.74 . The list of parameters employed in the fit is presented in Table 1. Figure 2c compares an experimental spectrum to the predicted rotational spectrum as provided by our current theoretical model. As can be seen from Fig. 2c, the overall correspondence between experimental and theoretical spectra is very good. A slight inconsistency with intensity between predicted and observed spectra that may be visible for some strong lines is due to variations of source power and detector sensitivity.

The dataset of fitted transitions is presented in Table 2 where we provide quantum numbers for each level, followed by observed transition frequencies, experimental uncertainties and residuals from the fit. For the ground state together with the first and the second quanta of the torsional mode $\left(v_{18}=1\right)$, a list of their predicted transition frequencies up to $600 \mathrm{GHz}\left(J_{\max }=\right.$ $50)$, their associated uncertainty, strength $\left(\mu^{2} S\right)$, Einstein coefficients $\left(A_{\mathrm{ul}}\right)$, and their lower state energy $\left(E^{\prime \prime}\right)$ is collected in Table 3. Since no rotational lines of $v_{12}=1$ or $J$ higher than 50 are included in the actual fit, higher discrepancies could be expected. The complete version of Tables 2 and 3 is available at the CDS, and only part of the tables is presented here for illustration purposes. The rotational and vibrational partition functions at different temperatures are tabulated in Table 4. The 
Table 2. Transitions included in the fit of $v_{\mathrm{t}}=0,1$ states.

\begin{tabular}{rrrrrrrrrrr}
\hline \hline$m^{\prime a}$ & $J^{\prime}$ & $K_{\mathrm{a}}^{\prime}$ & $K_{\mathrm{c}}^{\prime}$ & $m^{\prime \prime a}$ & $J^{\prime \prime}$ & $K_{\mathrm{a}}^{\prime \prime}$ & $K_{\mathrm{c}}^{\prime}$ & $\begin{array}{c}\text { Freq. meas. } \\
(\mathrm{MHz})\end{array}$ & $\begin{array}{c}\text { Uncert. } \\
(\mathrm{MHz})\end{array}$ & $\begin{array}{r}\text { Obs.-cal. } \\
(\mathrm{MHz})\end{array}$ \\
\hline-2 & 19 & 10 & 10 & -2 & 18 & 10 & 9 & 164450.2470 & 0.0500 & 0.0258 \\
0 & 19 & 4 & 16 & 0 & 18 & 4 & 15 & 164453.2689 & 0.0500 & 0.0129 \\
1 & 19 & 4 & 15 & 1 & 18 & 4 & 14 & 164453.6215 & 0.0500 & -0.0132 \\
1 & 19 & 4 & 16 & 1 & 18 & 4 & 15 & 164462.9970 & 0.0500 & 0.0028 \\
0 & 19 & 4 & 15 & 0 & 18 & 4 & 14 & 164463.8299 & 0.0500 & -0.0093 \\
-2 & 19 & 4 & 16 & -2 & 18 & 4 & 15 & 164479.1550 & 0.0500 & -0.0171 \\
1 & 19 & 9 & 10 & 1 & 18 & 9 & 9 & 164489.9111 & 0.0500 & -0.0217 \\
1 & 19 & 9 & 11 & 1 & 18 & 9 & 10 & 164493.2535 & 0.0500 & -0.0358 \\
0 & 19 & 3 & 17 & 0 & 18 & 3 & 16 & 164493.5134 & 0.0500 & -0.0414 \\
-3 & 19 & 9 & 10 & -3 & 18 & 9 & 9 & 164501.8950 & 0.0500 & 0.0058 \\
-3 & 19 & 9 & 11 & -3 & 18 & 9 & 10 & 164501.8950 & 0.0500 & 0.0058 \\
-2 & 19 & 13 & 6 & -2 & 18 & 13 & 5 & 164511.8920 & 0.1000 & 0.0078 \\
\hline
\end{tabular}

Notes. The complete table is available at the CDS. ${ }^{(a)}$ RAM36 code uses the free rotor quantum number $m$ for labeling torsional energy levels (Ilyushin et al. 2010; Belloche et al. 2017). For a molecule with three-fold barrier the $A$ and $E$ symmetry levels are labeled respectively as $m=0$, and $m=1$ for the ground state, as $m=-3$ and $m=-2$ for the first excited torsional state and as $m=3$ and $m=4$ for the second.

Table 3. Transitions of $v_{18}=0,1$ states predicted from the fit.

\begin{tabular}{rrrrrrrrrrrrrr}
\hline \hline$m^{\prime}$ & $J^{\prime}$ & $K_{\mathrm{a}}^{\prime}$ & $K_{\mathrm{c}}^{\prime}$ & $m^{\prime \prime}$ & $J^{\prime \prime}$ & $K_{\mathrm{a}}^{\prime \prime}$ & $K_{\mathrm{c}}^{\prime}$ & $\begin{array}{c}\text { Freq. calcu. } \\
(\mathrm{MHz})\end{array}$ & $\begin{array}{l}\text { Uncert. } \\
(\mathrm{MHz})\end{array}$ & $\begin{array}{c}\mu^{2} S \\
\left(\mathrm{D}^{2}\right)\end{array}$ & $A_{\mathrm{ul}}$ & $\begin{array}{c}E^{\prime \prime} \\
\left(\mathrm{cm}^{-1}\right)\end{array}$ \\
\hline 1 & 18 & 3 & 16 & 1 & 18 & 2 & 17 & 175474.6370 & 0.0447 & 2.516 & $4.276 \times 10^{-6}$ & 125.8268 \\
1 & 18 & 3 & 15 & 1 & 18 & 2 & 16 & 163222.1497 & 0.0356 & 2.652 & $3.628 \times 10^{-6}$ & 126.2704 \\
1 & 18 & 4 & 15 & 1 & 18 & 3 & 16 & 239680.0703 & 0.0332 & 2.291 & $9.923 \times 10^{-6}$ & 131.6800 \\
0 & 19 & 4 & 15 & 0 & 18 & 4 & 14 & 164463.8408 & 0.0051 & 107.951 & $1.433 \times 10^{-4}$ & 139.6885 \\
0 & 19 & 5 & 14 & 0 & 18 & 5 & 13 & 164400.9792 & 0.0052 & 105.132 & $1.394 \times 10^{-4}$ & 149.9849 \\
0 & 19 & 6 & 14 & 0 & 18 & 6 & 13 & 164391.1518 & 0.0055 & 101.686 & $1.348 \times 10^{-4}$ & 162.5623 \\
-3 & 19 & 5 & 14 & -3 & 18 & 5 & 13 & 164342.2552 & 0.0075 & 105.130 & $1.393 \times 10^{-4}$ & 286.5487 \\
-3 & 19 & 5 & 15 & -3 & 18 & 5 & 14 & 164342.0609 & 0.0075 & 105.130 & $1.393 \times 10^{-4}$ & 286.5487 \\
-3 & 19 & 6 & 13 & -3 & 18 & 6 & 12 & 164381.3368 & 0.0073 & 101.714 & $1.348 \times 10^{-4}$ & 298.8926 \\
-2 & 18 & 11 & 7 & -2 & 17 & 11 & 6 & 155675.6374 & 0.0139 & 66.989 & $7.951 \times 10^{-5}$ & 390.2943 \\
-2 & 18 & 12 & 7 & -2 & 17 & 12 & 6 & 156005.6329 & 0.0153 & 59.457 & $7.102 \times 10^{-5}$ & 415.3586 \\
-2 & 18 & 12 & 6 & -2 & 17 & 12 & 5 & 155754.0284 & 0.0192 & 59.399 & $7.060 \times 10^{-5}$ & 416.0377 \\
\hline
\end{tabular}

Notes. The complete table is available at the CDS.

vibrational one is calculated as $Q_{\mathrm{v}}=\prod^{\mathrm{v}}\left(1-\mathrm{e}^{-E_{\mathrm{v}} / K_{\mathrm{B}} T}\right)^{-d_{\mathrm{v}}}$ (see Eq. (3.60) from Gordy \& Cook 1984), where we considered the values of $v_{18}=1, v_{18}=2$ and $v_{12}=1$. The rotational partition function is evaluated by summation over all ground-state levels predicted from the fit up to $J=50$. From the values of Table 4, a significant contribution of the vibrational excited states to the partition function is expected at temperatures from $\sim 100 \mathrm{~K}$.

Direct comparison of the results obtained in this study with the results of Bak et al. $(1962,1966)$ is not obvious owing to the two different methods used and significantly different datasets. As has been pointed out previously, in the present study, the transitions involving levels with low values of $J$ and $K_{\mathrm{a}}$ quantum numbers from the studies of Bak et al. $(1962,1966)$ were refit to provide initial predictions. The results of this fit and several structural parameters related to the methyl torsion are presented in Table A.2. We also present in Table A.2 the same parameters issued from the global fit, and from ab initio calculations of the present study in order to provide a point of comparison. The rotational constants from ab initio calculations are given in the rho axis system for consistency. In general, the values obtained from this work are in agreement to those ab initio and those from
Table 4. Rotational and vibrational partition function for methyl ketene at different temperatures.

\begin{tabular}{ccc}
\hline \hline$T / K$ & $Q_{\mathrm{r}}$ & $Q_{\mathrm{v}}$ \\
\hline 300 & 53908.5 & 4.80 \\
225 & 38388.0 & 2.97 \\
150 & 22449.9 & 1.77 \\
75 & 8163.3 & 1.11 \\
37.5 & 2888.3 & 1.01 \\
18.75 & 1022.1 & 1.00 \\
9.375 & 362.3 & 1.00 \\
\hline
\end{tabular}

Notes. See end of Sect. 4 for further details.

the literature. We calculated the value of the methyl top moment of inertia $I_{\alpha}$ independently from $F$ and from the $\rho$ parameters (see equations in Table A.2 footnotes) to further check the consistency of the results of the global fit. In the rigid rotor approximation, the $I_{\alpha}$ values should be equal (Ilyushin 2011). Such an approximation is suitable for vibrational ground-state levels at low $J$ and $K_{\mathrm{a}}$ quantum numbers, as it is the case of the assigned 
Table 5. Physical parameters of the considered cloud cores.

\begin{tabular}{|c|c|c|c|c|c|c|c|c|}
\hline Source & $\begin{array}{l}\text { Coordinates } \\
(\mathrm{J} 2000.0)\end{array}$ & $\begin{array}{c}\mathrm{HPBW}^{a} \\
\quad\left({ }^{\prime \prime}\right)\end{array}$ & $\begin{array}{c}\text { Frequencies }^{b} \\
(\mathrm{GHz})\end{array}$ & $\begin{array}{c}v_{\mathrm{LSR}} \\
\left(\mathrm{km} \mathrm{s}^{-1}\right)\end{array}$ & $\begin{array}{l}\Delta v_{\mathrm{FWHM}} \\
\left(\mathrm{km} \mathrm{s}^{-1}\right)\end{array}$ & $\begin{array}{c}d_{\text {sou }} \\
\left({ }^{\prime \prime}\right)\end{array}$ & $\begin{array}{l}T_{\text {rot }} \\
(\mathrm{K})\end{array}$ & $\begin{array}{c}N\left(\mathrm{CH}_{3} \mathrm{CHCO}\right) \\
\times 10^{14}\left(\mathrm{~cm}^{-2}\right) \\
\end{array}$ \\
\hline $\begin{array}{l}\text { Orion KL } \\
\text { (IRAM } 30 \mathrm{~m} \text { ) }\end{array}$ & $\begin{array}{c}\alpha=5^{\mathrm{h}} 35^{\mathrm{m}} 14.5^{\mathrm{s}} \\
\delta=-05^{\circ} 22^{\prime} 30.0^{\prime \prime}\end{array}$ & $30-8$ & $80-307$ & 8 & 3 & 5 & 150 & $\leq(5.0 \pm 2.5)$ \\
\hline $\begin{array}{l}\text { Orion KL } \\
\text { (ALMA SV) } \\
\text { Hot core }\end{array}$ & $\begin{array}{c}\alpha=05^{\mathrm{h}} 35^{\mathrm{m}} 14.5^{\mathrm{s}} \\
\delta=-05^{\circ} 22^{\prime} 32.5^{\prime \prime}\end{array}$ & $\sim 1.9 \times 1.4$ & $213.7-246.7$ & 8 & 3 & 3 & 150 & $\leq(10 \pm 5)$ \\
\hline $\begin{array}{l}\text { Orion KL } \\
\text { (ALMA SV) } \\
\text { Compact ridge }\end{array}$ & $\begin{array}{c}\alpha=05^{\mathrm{h}} 35^{\mathrm{m}} 14.1^{\mathrm{s}} \\
\delta=-05^{\circ} 22^{\prime} 36.9^{\prime \prime}\end{array}$ & $\sim 1.9 \times 1.4$ & $213.7-246.7$ & 7.5 & 2 & 3 & 100 & $\leq(3.0 \pm 1.5)$ \\
\hline $\begin{array}{l}\text { Orion KL } \\
\text { (ALMA SV) } \\
\text { MM4 }\end{array}$ & $\begin{array}{c}\alpha=05^{\mathrm{h}} 35^{\mathrm{m}} 14.2^{\mathrm{s}} \\
\delta=-05^{\circ} 22^{\prime} 31.1^{\prime \prime}\end{array}$ & $\sim 1.9 \times 1.4$ & $213.7-246.7$ & $\begin{array}{l}3 \\
5\end{array}$ & $\begin{array}{l}3 \\
8\end{array}$ & $\begin{array}{l}3 \\
3\end{array}$ & $\begin{array}{l}150 \\
150\end{array}$ & $\begin{array}{l}\leq(5.0 \pm 2.5) \\
\leq(2.0 \pm 1.0)\end{array}$ \\
\hline Sgr B2(N) & $\begin{array}{c}\text { (IRAM 30 m) } \\
\alpha=17^{\mathrm{h}} 47^{\mathrm{m}} 20.0^{\mathrm{s}} \\
\delta=-28^{\circ} 22^{\prime} 19.0^{\prime \prime} \\
(\mathrm{GBT} 100 \mathrm{~m}) \\
\alpha=17^{\mathrm{h}} 47^{\mathrm{m}} 19.8^{\mathrm{s}} \\
\delta=-28^{\circ} 22^{\prime} 17.0^{\prime \prime}\end{array}$ & $30-21$ & $80-115.5$ & $\begin{array}{l}63 \\
72\end{array}$ & $\begin{array}{l}9 \\
8\end{array}$ & $\begin{array}{l}3 \\
3\end{array}$ & $\begin{array}{l}100 \\
100\end{array}$ & $\begin{array}{l}\leq(100 \pm 50) \\
\leq(100 \pm 50)\end{array}$ \\
\hline $\begin{array}{l}\text { B1-b } \\
\text { (IRAM } 30 \mathrm{~m} \text { ) }\end{array}$ & $\begin{array}{l}\alpha=03^{\mathrm{h}} 33^{\mathrm{m}} 20.0^{\mathrm{s}} \\
\delta=31^{\circ} 07^{\prime} 34.0^{\prime \prime}\end{array}$ & $30-21$ & $80-115.5$ & 6.7 & 0.7 & 60 & 12 & $\leq(0.010 \pm 0.005)$ \\
\hline $\begin{array}{l}\text { TMC-1 } \\
\text { (IRAM } 30 \mathrm{~m} \text { ) }\end{array}$ & $\begin{array}{c}\alpha=04^{\mathrm{h}} 41^{\mathrm{m}} 41.88^{\mathrm{s}} \\
\delta=25^{\circ} 41^{\prime} 27.0^{\prime \prime}\end{array}$ & $30-21$ & $80-115.5$ & 6.0 & 0.7 & 60 & 10 & $\leq(0.010 \pm 0.005)$ \\
\hline
\end{tabular}

Notes. ${ }^{(a)}$ HPBW (half power beam width) for observations with single dish telescope (IRAM $30 \mathrm{~m}$ and GBT $100 \mathrm{~m}$ ) and synthetic beam for the ALMA SV observations. ${ }^{(b)}$ Range of frequencies considered in the analysis.

transitions in previous studies (Bak et al. 1962, 1966). However, considering higher energy levels, including the first torsional state requires that we account for the distortion of the methyl top due to the rotational and torsional motion. Such distortion may be partially taken into account by the independent variation of $F$ and $\rho$. Consequently, $I_{\alpha}$ values calculated from $F$ and from $\rho$ may also differ in the case of a large dataset including excited torsional states. In the present study, the values of $I_{\alpha}(\rho)$ and $I_{\alpha}(F)$ are rather close with a relative error of about $1 \%$, and they are within the range of typical values of moments of inertia for the methyl group, $3.1 \mathrm{u}^{2}-3.3 \mathrm{u}^{2}$. Therefore we may claim that the obtained set of parameters presented in Table 1 properly describes the torsional-rotational problem for methyl ketene molecule. We also point out the improved accuracy of the main torsional-rotational parameters obtained in the present study, which also contributed to the accurate calculation of predicted spectra that were further used for radio astronomical observations.

\section{Radio-astronomical observations}

Thanks to the improved frequency predictions provided by the present work, we have carried out a rigorous search for methyl ketene in space. Although none of the $\mathrm{C}_{3} \mathrm{H}_{4} \mathrm{O}$ isomers have been unambiguously identified in space, the ubiquity of acetaldehyde $\left(\mathrm{CH}_{3} \mathrm{CHO}\right)$ in starless cores and star-forming regions (see, e.g., Ikeda et al. 2001; Kaifu et al. 2004; Cernicharo et al. 2012; Belloche et al. 2013) encourages us to search for methyl ketene $\left(\mathrm{CH}_{3} \mathrm{CHCO}\right)$ in those environments. We focused on the two most studied high-mass star-forming regions, Orion KL and Sagittarius (Sgr B2), on a starless core in Taurus Molecular Cloud (TMC-1), and on the cold dark cloud Barnard 1 (B1-b). We used
Table 6. Column density ratios.

\begin{tabular}{lcc}
\hline \hline Source & $\begin{array}{c}N\left(\mathrm{CH}_{3} \mathrm{CHO}\right)^{a} \\
\left(\mathrm{~cm}^{-2}\right)\end{array}$ & $\begin{array}{c}N\left(\mathrm{CH}_{3} \mathrm{CHO}\right)^{a} \\
N\left(\mathrm{CH}_{3} \mathrm{CHCO}\right)^{a}\end{array}$ \\
\hline Orion KL (HC) & $1.6 \times 10^{15 b}$ & $\geq 1.6$ \\
Orion KL (MM4) $^{15 b}$ & $1.4 \times 10^{15 b}$ & $\geq 2.0$ \\
Sgr B2 $^{a}$ & $2.2 \times 10^{17 c}$ & $\geq 11$ \\
B1-b & $1.5 \times 10^{12 d}$ & $\geq 1.5$ \\
\hline
\end{tabular}

Notes. ${ }^{(a)}$ Sum of the different spectral components. ${ }^{(b)}$ Cernicharo et al. (2016). ${ }^{(c)}$ Belloche et al. (2013). ${ }^{(d)}$ Cernicharo et al. (2012).

the MADEX code (Cernicharo 2012) to exploit the spectroscopic parameters presented in this work and to derive the synthetic spectrum of this species (assuming local thermodynamic equilibrium) according to the physical parameters of the source (see below) collected in Table 5. The column density was the only free parameter for these models. Corrections for beam dilution were applied to each line depending on its frequency.

Orion $K L$. Data from the Atacama Large Millimeter/submillimeter Array (ALMA) interferometer (Science Verification, SV) and the Institut de Radioastronomie Millimétrique (IRAM) $30 \mathrm{~m}$ telescope (Tercero et al. 2010, 2015) have been explored to search for $\mathrm{CH}_{3} \mathrm{CHCO}$. The ALMA SV data allow us to obtain the spectrum between 213.7 and $246.7 \mathrm{GHz}$ for different positions within the source characterized by different chemistry and physical parameters (see, e.g., Gong et al. 2015 and references therein). We distinguished (i) the hot core, a position in the middle of a clumpy structure where the emission 
ORION KL
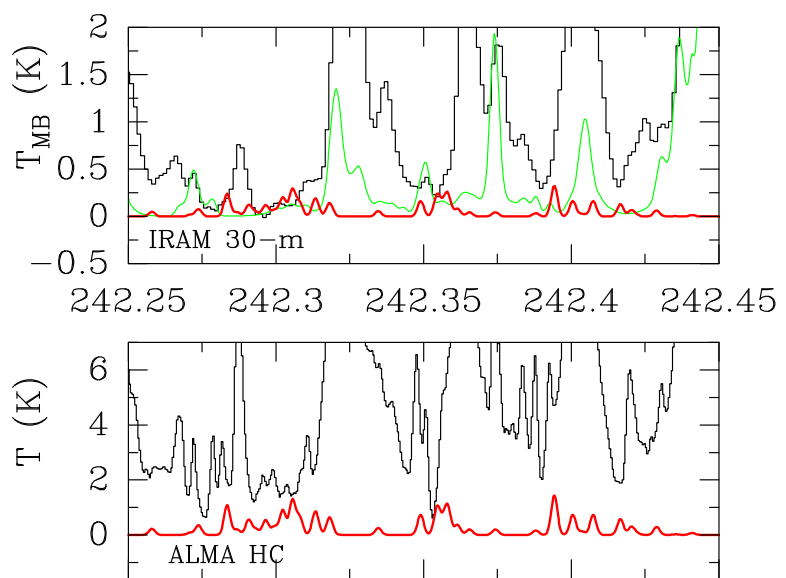

$\begin{array}{lllll}242.25 & 242.3 & 242.35 & 242.4 & 242.45\end{array}$
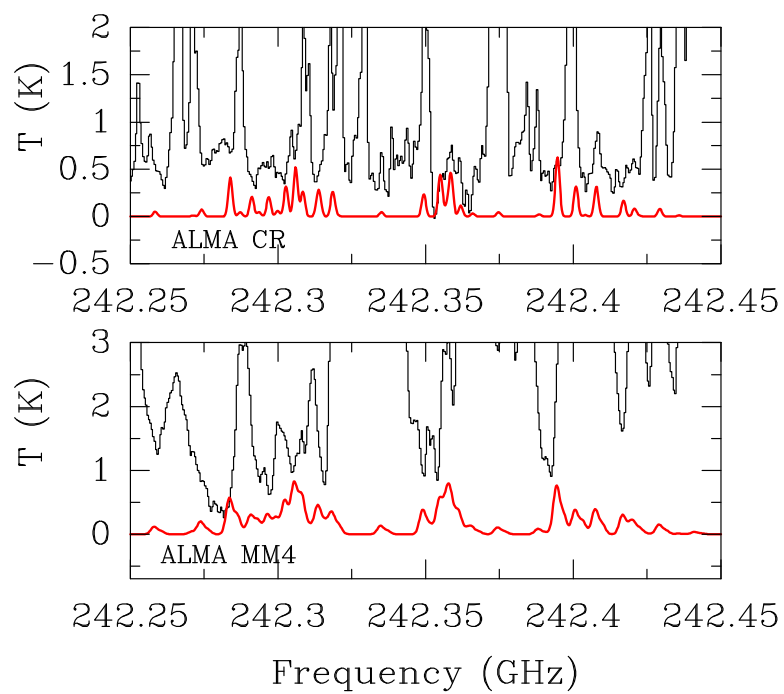
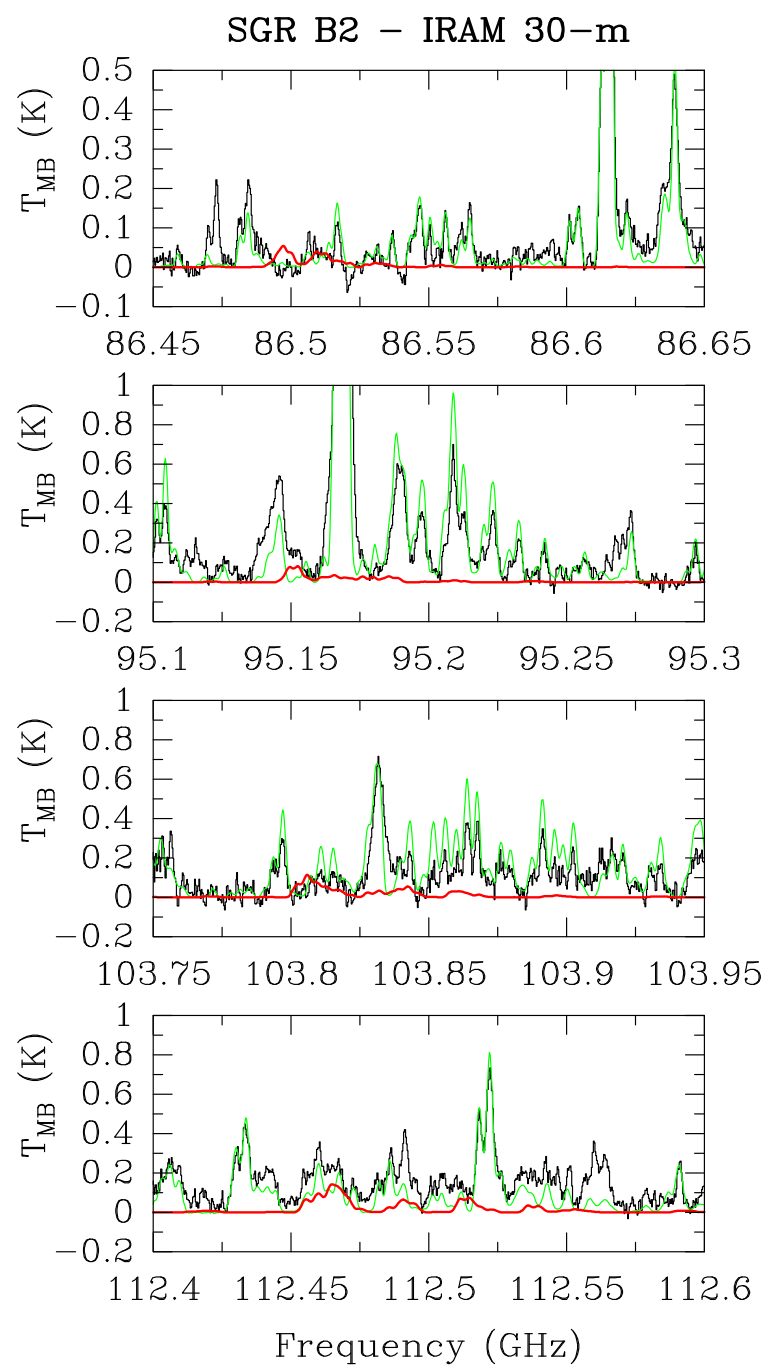

Fig. 4. Observed data of Orion KL and Sgr B2 taken with the IRAM 30-m telescope and the ALMA interferometer (histogram black spectrum) together with the synthetic spectra obtained using the column densities given as upper limits in Table 5 (red thin curve). The synthetic total model for the $30 \mathrm{~m}$ data of Sgr B2(N) (see Belloche et al. 2013) and Orion KL (see Cernicharo et al. 2016) is overlaid in green. A $v_{\mathrm{LSR}}$ of $+9.0 \mathrm{~km} \mathrm{~s}$ and $+64.0 \mathrm{~km} \mathrm{~s}^{-1}$ is assumed for Orion KL and Sgr B2, respectively.

peaks of ethylene glycol $\left(\mathrm{HOCH}_{2} \mathrm{CH}_{2} \mathrm{OH}\right.$, Brouillet et al. 2015), acetic acid $\left(\mathrm{CH}_{3} \mathrm{COOH}\right.$, Cernicharo et al. 2016; Favre et al. 2017), and methyl isocyanate $\left(\mathrm{CH}_{3} \mathrm{NCO}\right.$, Cernicharo et al. 2016) are located; (ii) the compact ridge, located at the emission peak of methyl formate $\left(\mathrm{CH}_{3} \mathrm{OCOH}\right.$, Favre et al. 2011), dimethyl ether $\left(\mathrm{CH}_{3} \mathrm{OCH}_{3}\right.$, Brouillet et al. 2013), and ethyl methyl ether $\left(\mathrm{CH}_{3} \mathrm{CH}_{2} \mathrm{OCH}_{3}\right.$, Tercero et al. 2015); and (iii) the MM4 source (see Wu et al. 2014), a clump that concentrates a large fraction of the emission of methyl isocyanate (Cernicharo et al. 2016) and acetone $\left(\mathrm{CH}_{3} \mathrm{COCH}_{3}\right.$, Peng et al. 2013; Cernicharo et al. 2016) in the region. In addition, the IRAM $30 \mathrm{~m}$ data provide a very wide frequency band $(80-307 \mathrm{GHz})$ of the average spectrum of the region inside the telescope beam. To model the $\mathrm{CH}_{3} \mathrm{CHCO}$ emission in these components, we have adopted physical parameters according to those derived by Cernicharo et al. (2016) and Tercero et al. (2015). Figure 4 shows selected frequencies and positions of these data together with the model derived by MADEX that demonstrates the lack of $\mathrm{CH}_{3} \mathrm{CHCO}$ lines above the confusion limit of these data.

Sgr B2. We also searched for $\mathrm{CH}_{3} \mathrm{CHCO}$ in public data available for Sgr B2. We did not find this species above the detection limit of the data either in the PRIMOS survey ${ }^{1}$ (Neill et al. 2012) or in the IRAM $30 \mathrm{~m}$ data at $3 \mathrm{~mm}$ provided by Belloche et al. (2013). To estimate upper limits to the $\mathrm{CH}_{3} \mathrm{CHCO}$ column density in the region, we adopted the physical parameters derived by Belloche et al. (2013) for $\mathrm{CH}_{3} \mathrm{CHO}$ (see Table 5). Figure 4 shows the model provided by MADEX (red line) together with the IRAM $30 \mathrm{~m}$ data of Sgr B2(N). Interestingly, this model is consistent with the lack of methyl ketene lines above the detection limit of the PRIMOS data.

B1-b and TMC-1. Finally, we used the data presented in Cernicharo et al. (2012) to derive upper limits to the methyl ketene column density in dark clouds at different evolutionary stages (see Table 5).

Table 6 shows the $\mathrm{CH}_{3} \mathrm{CHO} / \mathrm{CH}_{3} \mathrm{CHCO}$ column density ratios in Orion $\mathrm{KL}, \mathrm{Sgr} \mathrm{B} 2$, and B1-b. It is worth noting that the lower limit ratios derived agree for the considered Orion cores and for B1, and they are extremely low ( 2). For Sgr

1 The 100m Green Bank Telescope (GBT) PRebiotic Interstellar MOlecule Survey covers a frequency band between 7 and $50 \mathrm{GHz}$. Access to the entire PRIMOS data set, specifics on the observing strategy, and overall frequency coverage information is available at http: //www.cv.nrao.edu/ aremijan/PRIMOS/. 
B2 we found also a moderate value, only $\sim 6$ times larger than that derived for Orion. Although the dipole moment along the $a$-axis is similar for both species, the $\mathrm{CH}_{3} \mathrm{CHCO}$ partition function is $\sim 2$ times larger (at $10-150 \mathrm{~K}$ ) than that of $\mathrm{CH}_{3} \mathrm{CHO}$. As a result, the $\mathrm{CH}_{3} \mathrm{CHCO}$ lines appear weaker than those of $\mathrm{CH}_{3} \mathrm{CHO}$ assuming similar abundances. Therefore, it is reasonable to suggest that the derived lower limit is far from the real abundance ratio between these species in the considered regions. Achieving higher sensitivity of the data with the current generation of telescopes is crucial to determine a more realistic value of the $\mathrm{CH}_{3} \mathrm{CHO} / \mathrm{CH}_{3} \mathrm{CHCO}$ column density ratio.

\section{Conclusion}

Our theoretical calculation shows that methyl ketene (mk) is the $\mathrm{C}_{3} \mathrm{H}_{4} \mathrm{O}$ isomer with the closest energy to the most stable one, propenal (ap) $\left(\Delta \mathrm{E}_{\mathrm{ap}-\mathrm{mk}}=2.8 \mathrm{~kJ} \mathrm{~mol}^{-1}\right.$ at $\operatorname{CCSD}(\mathrm{T}) /$ aug-cc-pVTZ level of theory). Three transitions of the latter have been already detected towards Sgr B2 (Hollis et al. 2004). The closeness in energy of both isomers should make methyl ketene as good a candidate as anti-propenal for detection in the ISM. The millimeter-wave spectrum of this molecule has been analyzed in the $50-330 \mathrm{GHz}$ frequency range in order to provide accurate rotational parameters that could allow its detection. The internal rotation of the $\mathrm{CH}_{3}$ group was analyzed employing the rho-axis method and the program RAM36 (Ilyushin et al. 2010). Notwithstanding the complexity of the analysis, more than 3000 transitions were assigned to the ground and first torsional state of methyl ketene. The height of the internal rotation barrier was found to be $429.37(11) \mathrm{cm}^{-1}$ and the $\rho$ value $0.194354(13)$. Both values are intermediate between acetic acid with lower values of $\rho$ and $V_{3}$ (Ilyushin et al. 2013) and acetaldehyde with higher ones (Smirnov et al. 2014). These parameters were employed to unsuccessfully search for its presence in star-forming regions at different evolutionary stages: Orion KL, Sgr B2, TMC-1, and B1-b. Higher sensitivity of the data is required to achieve a realistic abundance ratio between acetaldehyde and methyl ketene in the ISM.

Acknowledgements. The research leading to these results has received funding from the French Programme National "Physique et Chimie du Milieu Interstellaire", and from the ANR-13-BS05-0008 IMOLABS of the French Agence Nationale de la Recherche. B.T. and J.C. thank funding support from Spanish MINECO (grants AYA2012-32032, AYA2016-75066-C2-1-P, and CSD200900038) and from the European Research Council (grant ERC-2013-SyG 610256, NANOCOSMOS). J.-C. G. thanks the Centre National d'Etudes Spatiales (CNES) for financial support.

\section{References}

Bak, B., Christensen, D., Christiansen, J., Hansen-Nygaard, L., \& RastrupAndersen, J. 1962, Spectrochim. Acta, 18, 1421

Bak, B., Christensen, J. J., Kunstmann, K. A. J., Nygaard, L., \& RastrupAndersen, J. 1966, J. Chem. Phys., 45, 883
Belloche, A., Müller, H. S. P., Menten, K. M., Schilke, P., \& Comito, C. 2013, A\&A, 559, A47

Belloche, A., Meshcheryakov, A., \& Garrod, R. 2017, A\&A, 601, A49

Bottinelli, S., Ceccarelli, C., Williams, J. P., \& Lefloch, B. 2007, A\&A, 463, 601

Brouillet, N., Despois, D., \& Baudry, A. 2013, A\&A, 550, A46

Brouillet, N., Despois, D., \& Lu, X. H. 2015, A\&A, 576, A129

Cernicharo, J. 2012, in ECLA-2011: Proceedings of the European Conference on Laboratory Astrophysics, Laboratory Astrophysics and Astrochemistry in the Herschel/ALMA Era, 58, 251

Cernicharo, J., Marcelino, N., \& Roueff, E. 2012, ApJ, 759, L43

Cernicharo, J., Kisiel, Z., \& Tercero, B. 2016, A\&A, 587, L4

Daly, A. M., Bermudez, C., Kolesnikova, L., \& Alonso, J. L. 2015, ApJS, 218, 30

Endres, C. P., Schlemmer, S., Schilke, P., Stutzki, J., \& Müller, H. S. 2016, J. Mol. Spectr., 327, 95

Favre, C., Despois, D., \& Brouillet, N. 2011, A\&A, 532, A32

Favre, C., Pagani, L., \& Goldsmith, P. F. 2017, A\&A, 604, L2

Frisch, M. J., Trucks, G. W., \& Schlegel, H. 2016, Gaussian09, Revision D. 01 (Wallingford, CT: Gaussian. Inc)

Gong, Y., Henkel, C., \& Thorwirth, S. 2015, A\&A, 581, A48

Gordy, W., \& Cook, R. 1984, Microwave Molecular Spectra (New York: Wiley) Herbst, E., \& van Dishoeck, E. F. 2009, ARA\&A, 47, 427

Hollis, J. M., Jewell, P. R., Lovas, F. J., Remijan, A. J., \& Mollendal, H. 2004, ApJ, 610, L21

Hollis, J. M., Anthony, J. R., Jewell, P. R., \& Lovas, F. J. 2006a, ApJ, 642, 933

Hollis, J. M., Lovas, F. J., \& Remijan, A. J. 2006b, ApJ, 643, L25

Ikeda, M., Ohishi, M., \& Nummelin, A. 2001, ApJ, 560, 792

Ilyushin, V., Kryvda, A., \& Alekseev, E. 2009, J. Mol. Spectr., 255, 32

Ilyushin, V. V. 2011, Radio Physics and Radio Astronomy, 16, 414

Ilyushin, V. V., Kisiel, Z., Pszczókowski, L., Mäder, H., \& Hougen, J. T. 2010, J. Mol. Spectr., 259, 26

Ilyushin, V. V., Endres, C. P., Lewen, F., Schlemmer, S., \& Drouin, B. J. 2013, J. Mol. Spectr., 290, 31

Jiménez-Serra, I., Vasyunin, A. I., \& Caselli, P. 2016, ApJ, 830, L6

Jørgensen, J. K., van der Wiel, M. H. D., \& Coutens, A. 2016, A\&A, 595, A117

Kaifu, N., Ohishi, M., \& Kawaguchi, K. 2004, PASJ, 56, 69

Karton, A., \& Talbi, D. 2014, Chem. Phys., 436-437, 22

Kleiner, I. 2010, J. Mol. Spectr., 260, 1

Lattelais, M., Pauzat, F., Ellinger, Y., \& Ceccarelli, C. 2009, ApJ, 696, L133

Lattelais, M., Pauzat, F., Ellinger, Y., \& Ceccarelli, C. 2010, A\&A, 519, A30

Lattelais, M., Bertin, M., \& Mokrane, H. 2011, A\&A, 532, A12

McGuire, B. A., Burkhardt, A. M., \& Kalenskii, S. 2018, Science, 359, 202

Neill, J. L., Muckle, M. T., \& Zaleski, D. P. 2012, ApJ, 755, 153

Peng, T. C., Despois, D., \& Brouillet, N. 2013, A\&A, 554, A78

Quan, D., \& Herbst, E. 2007, A\&A, 474, 521

Remijan, A., Shiao, Y. S., Friedel, D. N., Meier, D. S., \& Snyder, L. E. 2004, ApJ, 617, 384

Requena-Torres, M. A., Martín-Pintado, J., \& Rodríguez-Franco, A. 2006, A\&A, 455,971

Smirnov, I., Alekseev, E., Ilyushin, V., et al. 2014, J. Mol. Spectr., 295, 44

Tercero, B., Cernicharo, J., Pardo, J. R., \& Goicoechea, J. R. 2010, A\&A, 517, A96

Tercero, B., Kleiner, I., \& Cernicharo, J. 2013, ApJ, 770, L13

Tercero, B., Cernicharo, J., \& López, A. 2015, A\&A, 582, L1

Turner, B. E. 1977, ApJ, 213, L75

Werner, H. J., Knowles, P. J., \& Knizia, G. 2015, MOLPRO, version 2015.1, a package of ab initio programs

Winther, F., Meyer, S., \& Nicolaisen, F. M. 2002, J. Mol. Struct., 611, 9

Wu, Y., Liu, T., \& Qin, S. L. 2014, ApJ, 791, 123

Xu, L.-H., Fisher, J., Lees, R., et al. 2008, J. Mol. Spectr., 251, 305

Zakharenko, O., Motiyenko, R. A., Margulès, L., \& Huet, T. 2015, J. Mol. Spectr., 317, 41 


\section{Appendix A: Complementary tables}

Table A.1. Ab initio calculations at MP2/aug-cc-pVTZ level of theory.

\begin{tabular}{lrllrr}
\hline \hline Parameters $^{a}$ & Value & Unit & Structure $^{b}$ & Value & Unit \\
\hline$A_{\text {PAM }}$ & 38445.435 & $\mathrm{MHz}$ & $\mathrm{R}(1,2)$ & 1.1643 & $\AA$ \\
$B_{\text {PAM }}$ & 4507.167 & $\mathrm{MHz}$ & $\mathrm{R}(1,3)$ & 1.3141 & $\AA$ \\
$C_{\text {PAM }}$ & 4137.882 & $\mathrm{MHz}$ & $\mathrm{R}(3,4)$ & 1.0800 & $\AA$ \\
& & & $\mathrm{R}(3,5)$ & 1.5102 & $\AA$ \\
$A_{\text {RAM }}$ & 38149.156 & $\mathrm{MHz}$ & $\mathrm{R}(5,6)$ & 1.0907 & $\AA$ \\
$B_{\mathrm{RAM}}$ & 4803.446 & $\mathrm{MHz}$ & $\mathrm{R}(5,7)$ & 1.0907 & $\AA$ \\
$C_{\mathrm{RAM}}$ & 4137.882 & $\mathrm{MHz}$ & $\mathrm{R}(5,8)$ & 1.0895 & $\AA$ \\
$D_{\mathrm{ab}}$ & -3157.121 & $\mathrm{MHz}$ & $\mathrm{A}(1,3,4)$ & 115.76 & $\circ$ \\
& & & $\mathrm{A}(1,3,5)$ & 122.45 & $\circ$ \\
$\theta$ & 5.36 & $\circ$ & $\mathrm{A}(4,3,5)$ & 121.79 & $\circ$ \\
$\rho$ & 0.191 & & $\mathrm{~A}(3,5,6)$ & 110.97 & $\circ$ \\
$V_{3}$ & 424.709 & $\mathrm{~cm}-1$ & $\mathrm{~A}(3,5,7)$ & 110.97 & $\circ$ \\
$F$ & 187.903 & $\mathrm{GHz}$ & $\mathrm{A}(3,5,8)$ & 110.94 & $\circ$ \\
$s$ & 30.12 & $\mathrm{Unitless}$ & $\mathrm{A}(6,5,7)$ & 108.18 & $\circ$ \\
& & & $\mathrm{A}(6,5,8)$ & 107.82 & $\circ$ \\
$\Delta_{\mathrm{J}}$ & 2.618 & $\mathrm{kHz}$ & $\mathrm{A}(7,5,8)$ & 107.82 & $\circ$ \\
$\Delta_{\mathrm{IK}}$ & 1860.255 & $\mathrm{kHz}$ & $\mathrm{D}(1,3,5,6)$ & 119.84 & $\circ$ \\
$\Delta_{\mathrm{K}}$ & -91.960 & $\mathrm{kHz}$ & $\mathrm{D}(1,3,5,7)$ & -119.84 & $\circ$ \\
$\delta_{\mathrm{J}}$ & 0.553 & $\mathrm{kHz}$ & $\mathrm{D}(1,3,5,8)$ & 0.00 & $\circ$ \\
$\delta_{\mathrm{K}}$ & 5.577 & $\mathrm{kHz}$ & $\mathrm{D}(4,3,5,6)$ & -60.16 & $\circ$ \\
& & & $\mathrm{D}(4,3,5,7)$ & 60.15 & $\circ$ \\
$\mu_{\mathrm{a}(\mathrm{RAM})}$ & 1.65 & $\mathrm{D}$ & $\mathrm{D}(4,3,5,8)$ & 180.00 & $\circ$ \\
$\mu_{\mathrm{b}(\mathrm{RAM})}$ & 0.33 & $\mathrm{D}$ & & & \\
\hline
\end{tabular}

Notes. ${ }^{(a)} A_{\mathrm{PAM}}, B_{\mathrm{PAM}}, C_{\mathrm{PAM}}$ are the rotational parameters in the principal axis frame, $A_{\mathrm{RAM}}, B_{\mathrm{RAM}}, C_{\mathrm{RAM}}$ and $D_{\mathrm{ab}}$ are the rotational parameters in the rho frame, $V_{3}$ is the heigh of the tortional barrier, $\rho, s, F$ and $\theta_{\text {RAM }}$ are defined in the caption of Table A.2, $F$ is the reduced rotational constant, $\Delta_{\mathcal{J}}, \Delta_{\mathcal{J} \mathcal{K}}, \Delta_{\mathcal{K}}, \delta_{\mathcal{J}}$ and $\delta_{\mathcal{K}}$ are the centrifugal distortion constants and $\mu_{\mathrm{a}(\mathrm{PAM})}$ and $\mu_{\mathrm{b}(\mathrm{PAM})}$ are the dipole moment components in the $a$ and $b$ axes of the principal axis frame. ${ }^{(b)}$ Bond distances, bond angles and dihedral angles of methyl ketene. Labelling followed as in the figure of Table A.2.

Table A.2. Rotational constants and internal rotation parameters in the $\rho$-axes-frame.

\begin{tabular}{llrrrr}
\hline Axes and angles $^{a}$ & Parameters $^{b}$ & Our fit from refs. $^{d}$ & MP2/aug-cc-pVTZ & Our work & Units \\
\hline & $A_{\mathrm{RAM}}$ & $38641 .(160)$ & 38149.2 & $38587.00(11)$ \\
$\mathrm{MHz}$ \\
\end{tabular}

Notes. ${ }^{(a)}$ Axis frames in methyl ketene under the $I^{r}$ representation: in black the principal axis frame and in red the rho-axis. In blue, it is indicated the internal rotation axis. ${ }^{(b)} A_{\mathrm{RAM}}, B_{\mathrm{RAM}}, C_{\mathrm{RAM}}$ are the rotational constants in the rho-axis-frame. $V_{3}$ is the height of the torsional barrier. $\rho$ is the norm of the $\rho$ vector described in Sect. 4 and represented in green in the figure. $F$ is the internal rotational constant defined by $F=h^{2} / 2 r I_{\alpha}$ where $r=1-\sum_{g} 2 \lambda_{g}^{2} I_{\alpha} / I_{g}$ and $\lambda_{g}$ the direction cosines mentioned in Sect. $4 . s$ is the reduced barrier parameter which is calculated as $s=4 V_{3} / 9 F . \delta$ is the angle between the internal rotation and the $a_{\mathrm{PAM}}$ axis, which is thus related to the direction cosines as $\cos (\delta)=\lambda_{\mathrm{a}} \cdot \theta_{\mathrm{RAM}}$ is the angle of frame rotation and can be calculated based on the $\rho$ projections: $\theta=\arctan \left(\rho_{x} / \rho_{y}\right)$. Finally, $I_{\alpha}$ is the reduced mass of the methyl top. ${ }^{(c)} I_{\alpha}$ is, in case of the ab initio parameters, fixed to 3.2 which is its average value for a methyl top in the calculation from the ab initio structure. In the other cases it is calculated from the RAM rotational constants and either the $\rho$ or $F$ values as follows (Ilyushin 2011): $\rho=I_{\alpha}\left(A_{\mathrm{RAM}} B_{\mathrm{RAM}}-D_{\mathrm{ab}}^{2}\right) / 0.5 \hbar^{2} \sqrt{B_{\mathrm{RAM}}^{2}+D_{\mathrm{ab}}^{2}}$ and $F=0.5 \hbar^{2}\left[I_{\alpha}-\left(I_{\alpha}^{2} B_{\mathrm{RAM}}\left(A_{\mathrm{RAM}} B_{\mathrm{RAM}}-D_{\mathrm{ab}}^{2}\right) / 0.5 \hbar^{2}\left(B_{\mathrm{RAM}}^{2}+D_{\mathrm{ab}}^{2}\right)\right)\right]^{-1} \cdot{ }^{(d)}$ Values obtained employing our RAM36 fit of the transitions from Bak et al. (1962) and the methyl ketene experimental structure from Bak et al. (1966). 